\title{
Use of Fuzzy Reasoning in the Simulation of Risk Events in Business Processes
}

\author{
Paul Taylor, Jesús Jimenez Godino, and Basim Majeed \\ Intelligent Systems Research Centre, \\ British Telecommunications plc, \\ pp. 12, $1^{\text {st }}$ Floor, Orion Building, Adastral Park, Martlesham Heath, IPSWICH, UK, IP5 3RE \\ E-mail: \{paul.n.taylor, jesus.jimenezgodino, basim.majeed\}@bt.com
}

\section{KEYWORDS}

Simulation, Risk, Business Process, Fuzzy Logic, KPI, KRI.

\begin{abstract}
The current drive towards Service Oriented Architecture (SOA) and Business Process Execution Language (BPEL) in enterprises will increase dependency on efficient businesses processes. In the current competitive environment, process efficiency gains are seen as a crucial factor for business success. However it is not sufficient to design a process that works well under normal conditions. Risk analysis and mitigation is an important activity that should be tackled systematically during process design and improvement. The process designer's job has thus become particularly complex, requiring tools that combine traditional business process management with operational risk analysis.

In this paper we introduce a simulation environment that has been developed within British Telecommunications plc to simulate business process performance. The simulator incorporates a facility to simulate arbitrary risk effects on the performance of the process. Since risk analysis typically deals with qualitative values such as "high probability risk" or "low impact risk", measuring key risk indicators (KRIs) can be difficult. The simulator allows the process designer to formulate a fuzzy system of rules to define how risk is measured; these allow the user to produce KRIs that utilise the qualitative risk knowledge in addition to the ability to derive quantitative risk measures should they be needed.
\end{abstract}

\section{INTRODUCTION}

The increasingly process driven nature of businesses and the increasing interest in Service Oriented Architecture (SOA) and Business Process Orchestration technologies such as Business Process Execution Language (BPEL) in IT Departments in many companies mean it is imperative that each business process be as efficient as possible, both in terms of the systems that are invoked in the course of process execution and in terms of the human tasks that are required to ensure a favourable result.

In this paper we showcase some of the innovations that are currently being developed within British
Telecommunications plc (BT) to drive efficiencies within business processes and also showcase some of the tools that are being developed to aid process designers in designing and re-engineering efficient business processes.

Our existing work on Real-Time Business Intelligence (RTBI) (Azvine et al. 2006) has emphasised the need for businesses to develop and use efficient business processes that are able to respond appropriately to internal and external factors that can affect the outcome or execution of the process.

To this end we have developed an extensible process simulation tool that allows Process Designers to simulate the execution of their business processes and gain insights into the outcome of those process in terms of the estimated value of specified Key Performance Indicators (KPI) and in terms of the throughput of the process through the estimation and visualisation of the queues for each activity.

As is discussed in the literature (Lambert 2006; Neiger 2006) good business processes should take into account the risks involved in carrying out the process and choices at design time should take into account the level of risk for each activity so the overall level of risk for the holistic process is minimised.

Our simulation tool builds upon plain discrete event simulation of business processes and includes facilities to utilise uncertainty and risk within the analysis that is performed including the ability to use of fuzzy logic to model risk.

The tool is targeted at process designers and process managers in order to provide some predictive analytic capability. This would allow stakeholders to recalculate Key Risk Indicators (KRI) quickly when considering adjustments to the process and additionally gain insights into the performance of the process both with and without the possibility of risk occurring during the process execution.

In this paper we next introduce some information about business process management (BPM) as a discipline and its links with risk analysis. The third section introduces the different features of the simulator. The fourth section provides a concrete example of how the tool is used giving examples of the insights that can be gained. The final section concludes the paper. 


\section{BUSINESS PROCESS MANAGEMENT \& RISK}

Business processes are defined by Davenport (1993) as a set of structured, measured activities across time and place, with a beginning, an end, and clearly identified inputs and outputs which are designed to produce a specified output for a particular customer or market. It is easy to see from this definition that most businesses can be seen as a collection of business processes. An efficient set of business processes is therefore essential to the efficient running of such a 'process driven' business.

Producing efficient business processes is not an easy task. One of the most important tools in process design and improvement is a simulation environment that can provide insight into the bottlenecks that could be encountered in the process, and also will allow the designer to see the effect of differing resource levels upon the defined process.

A number of articles in the literature mention the link between business processes and risk analysis (Lambert et al. 2006; Neiger et al. 2006; Jallow et al. 2007). The importance of analysing process risk led to this work which extends a basic process simulator with the ability to simulate the effect of risk events upon the process execution.

According to Frost et al. (2001) a risk is an uncertain future event which may have an effect upon the ability of an organisation to reach its objectives. Furthermore a risk consists of two attributes: first, a probability of occurrence and second, an effect.

Before the simulation approach discussed in this paper can begin it would be necessary for the risks involved in the process to be defined. The best approach would be to leverage a standard risk analysis process such as COSO (2004), for more information see Jallow et al. (2007).

Once risks to a process have been identified and assigned a probability and an effect, a corresponding risk can be defined within the simulator and will be used in the simulation of the process model when executed. These risk probabilities and risk effects are typically of a qualitative nature. This presents a challenge for the simulation of business processes and because risk analysis of a process is of limited value without knowledge of the business performance under the specified risk, it is important for the simulator to be able to express the performance of the process in values which can be easily understood by business users, that is to say quantitative values.

In the paper by Intaver (2005) a strong case is made for a combined quantitative and qualitative approach to risk management to aid project managers in accurate estimation of risks and effects. Zadeh (1994) argues that humans will typically prefer to reason about risks in a qualitative way. However since this reasoning is typically subjective it can be compromised by a number of other factors that may effect the quality of the results that are obtained. In addition the performance of business processes is typically measured in a quantitative rather than qualitative way and a quantitative answer that illustrates business performance is usually desirable.

Fuzzy logic allows for reasoning about quantitative measures in a qualitative way, and as such seems to be an ideal solution to the issues that we have described.

As such, we have implemented a simulation environment that allows the users of the system to define fuzzy logic rules to express the qualitative nature of risks to the process under test, and have the simulator return the performance of the business process in terms of KPIs that allow for easy comparison across simulation executions.

In addition to the advantages of fuzzy logic for reasoning about systems, it also allows the system to suppress small changes in those input values and therefore provide a more holistic view of the process and its simulation.

\section{PROCESS SIMULATOR}

The process simulator forms only one part of the RTBI framework that is being developed within BT Research \& Venturing. Specifically the simulator is built on top of the open-source JBPM stack and uses their JPDL workflow language to define processes. Both of these components are produced by JBoss, which is a division of Red Hat Middleware, LLC. (http://www.jboss.com).

The defined process is described to the system using the JBPM process designer, itself an extension to the Eclipse Framework (http://www.eclipse.org). Processes are represented as a series of opaque boxes (one per task) each with associated input and output attributes which can be defined by the user. In addition to allow the monitoring of task level attributes in the results of the simulation these output attributes can be mapped onto process output attributes.

\section{Simulation Approach}

The simulator is constructed of a number of highlevel components (Azvine et al. 2007):

- Event-Scheduler - This is used to fire events at the appropriate time within the simulators abstract time scale.

- Workflow System - This is used to ensure the execution of the process follows the process model

- Process Sources - These are used to generate new process instances which are then used as start points for new simulated processes.

- Task Execution Estimators - These estimate the outputs for each task.

- Resource Management System - This controls the assignment of actors to tasks.

Monitoring of a simulated process can be performed in a number of complementing ways within the simulator.

First, a KPI can be defined. These are most useful for monitoring the estimated performance of the process 
during the simulation itself. An example would be, the time taken between the placing of an order and the associated installation task being completed.

Second, a rule could be defined. Rules are used to trigger an action, or consequent, when a condition is met either at the end of a process execution or at specific time intervals (for globally applicable rules). For example, a process level rule could be triggered when an installation task passes the target date set by the Service Level Agreement covering that task. Alternatively a global rule could be triggered when the time spent waiting for an installation to occur exceeds the current average wait time. Another usage for rules would be to monitor regulatory compliance.

Third, a KRI could be defined. These are similar to KPIs in many ways however their purpose is different. Intuitively a KPI is intended to measure business performance however a KRI is intended to measure risk factors. The simulator allows KPIs and KRIs to be defined separately for simplicity.

The simulator uses a custom syntax to define KPIs, KRIs, rules, and risks. The syntax itself is reasonably intuitive and those extracts embedded in the text will be accompanied by a plain language explanation.

In a departure from traditional business intelligence products these monitoring criteria can be defined dynamically by business users without knowledge of the underlying semantics. This removes the time-lag that could occur if a new KPI, rule, or KRI is required.

Once these monitoring criteria are defined and the simulator initialised then the simulation can be executed. The simulation results are then stored in a database to allow analysis to be performed at any time between the end of the simulation and the time the simulator is reset.

The simulator also allows for dynamic adjustment of resource levels to allow the user to easily check the performance of the process with differing resource scenarios.

\section{Simulation of Risk}

The previous section describes the basic functionality of the simulator. If run as above, the simulator will not consider the effect that appropriate risks may have on the execution or outcome of a process or task. However it will have triggered the appropriate rules, and have available results for each of the KPI and KRIs that have been defined.

In order to consider risks within the analysis, each risk that is to be tested needs to be defined in a form the simulator can understand. This is accomplished using a graphical editor similar to that provided for KPI definition and rule definition. The user will need to provide the two components of a risk - the probability of occurrence, and the consequence of the risk occurring.

When the simulation is then executed, it will proceed as before until a task with an associated risk is to be executed, the simulation engine will then probabilistically decide if the risk would occur in that task instance and then factor in the consequences accordingly. By varying the consequences and probability the simulator can estimate how this business process will react to risk, and evaluate the KRIs and KPIs under these conditions.

In order to provide maximum flexibility, the simulator allows for the risks to be enabled and disabled for each simulation execution. This will allow additional analysis to discover the most debilitating risk in terms of effect on business performance which may be important for risk analysis. This sensitivity analysis is particularly important to identify areas that would benefit from process improvement. According to Papamichail \& Robertson (2004) an additional benefit, of sensitivity analysis can be to increase confidence in the results of risk analysis, and aid the identification of the best way to proceed when presented with a variety of options.

\section{Fuzzy Logic}

Fuzzy logic can be used in the definition of KPIs and KRIs. It allows us to reason with continuous criteria (e.g. temperature) in an intuitive way. This document cannot include a detailed introduction to fuzzy logic however interested readers are directed to the wide variety of literature on that topic for further information.

One key use of fuzzy logic, inside the simulator is through the keyword is in a statement like "@temperature is hot" this will obtain the value of temperature from the process outputs and consult the associated fuzzy class for the term hot. It will then compare these and return a truth value for temperature belonging to the class 'hot' between 0 and 1 with 0 being not contained at all and 1 being completely contained in the class 'hot'.

Inside the simulator fuzzy classes are created using a graphical editor in which the user is asked for the name of the set, the number of classes to be defined, the upper and lower bounds of values for this class, and the type (triangular, trapezoidal, bell-shaped, list) of function that models the transition between classes. The function can be adjusted by dragging the function curve into the desired position.

As well as testing membership with fuzzy logic, we can also use the simulator to derive values for KPI and KRIs using fuzzy systems.

For example a KRI could be defined with rules similar to those in Figure 1 to derive a result that represents a risk value based on the cost and duration of the job. Within the current implementation of the simulation environment a fuzzy system can contain as many or as few rules as are required to express the property. It is worth noting at this point that fuzzy systems can also be used to define KPIs in a similar way.

In addition to using fuzzy logic to derive compound values as above, the simulator can also derive and display the fuzzy distribution that results from the application of a fuzzy set. This can help to identify the 
risk profile of the process instances and could be an aid in identifying trends.

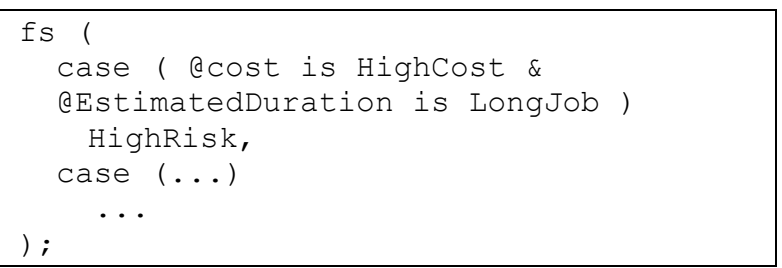

Figure 1: An example fuzzy definition

\section{EXAMPLE - CLIENT-ORDER PROCESS}

Now that the basics of the simulator have been presented this section will introduce an example of the simulator operation and of the results that can be expected.

The process that will be used is the following simple ordering process for computing services. When a client of the company places an order it is received by a member of the company's contact centre. The order is then classified to determine which type of work is required. One of the company's engineers then takes the job and completes it appropriately at the customer's premises.

A graphical depiction of the process is shown in Figure 2.

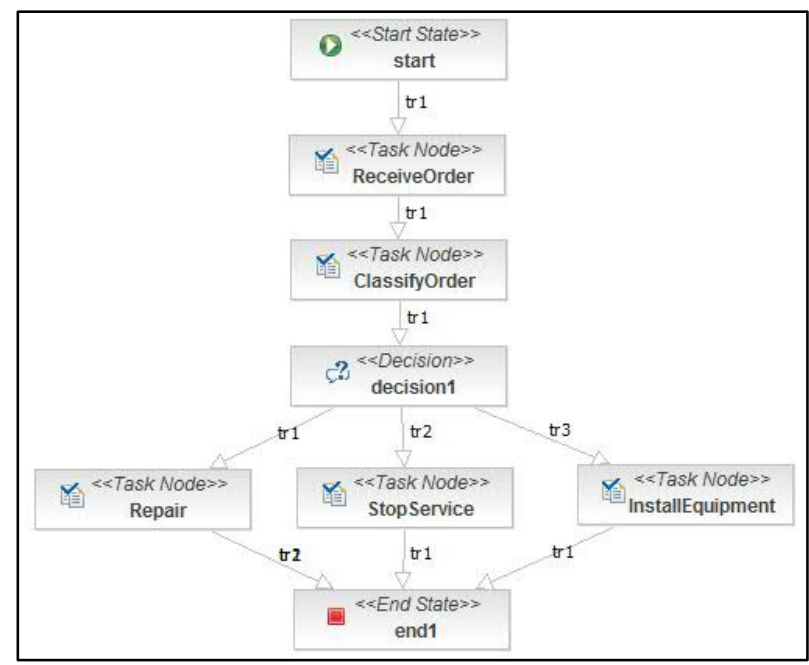

Figure 2: A Screenshot Showing the Process to Import

\section{Initialising the Simulator}

In order to simulate the process we first need to create the process with the editor. The process will need a single start node (which would be added ahead of ClassifyOrder) and a single end node (which is connected to all of the last 3 steps). Additionally an explicit decision node will need to be added between Classify Order and the lower activities to make the choice explicit. The process as it appears in the process designer is shown in Figure 2.

Once the simulator is loaded we can then import the process, set up the time parameters, and then complete the initialisation by setting a task assignment policy and process source. We assume from this point on that the source is configured to generate new processes at the rate to be tested, and an appropriate assignment strategy has been selected.

Once this is done we are free to define the KPIs, rules, KRIs, and risks that we would like to be simulated.

\section{Key Performance Indicators}

Consider that the business contains two types of employee who will take part in the process, OrderHandlers (initially 3) and Engineers (initially 3), and the company is interested in the overall end to end duration of the process which is typically called CycleTime. The definition of CycleTime in the monitor is very simple; we add a new KPI defined as: Qduration; (which is the duration of the process) and after the simulation is run we can request a report of expected performance on this measure. We also select to monitor the length of the queue for each activity during the simulation and after the simulation is complete.

Suppose we then simulate the process under these assumptions and find the CycleTime is growing as the simulation continues (as in Figure 3). This is clearly a cause for concern to the business. We can now check the queues to try and find an indication of which activity(s) are causing the issue.

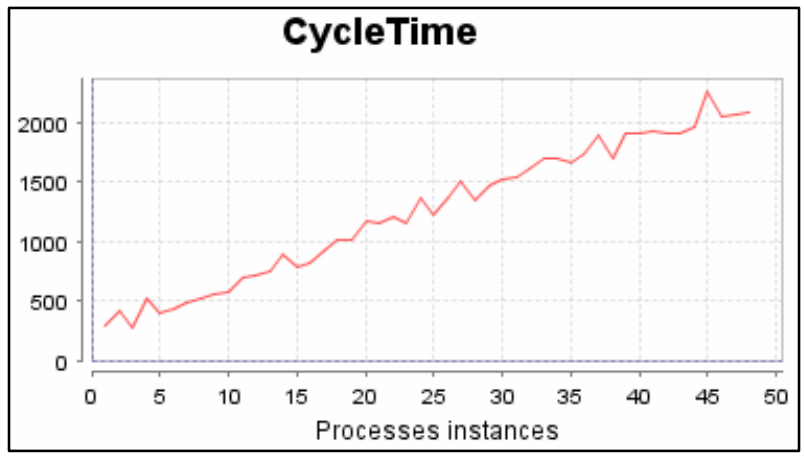

Figure 3: The CycleTime with 3 OrderHandlers

When checking the queues we find that both the ReceiveOrder and ClassifyOrder task queues grow in a similar way, but the queues for the engineer tasks are empty. From this we can conclude that changes need to be made to the order handling tasks to reduce the CycleTime. The process owner sees this data and would like to see how many additional staff in the OrderHandler role would be required to keep the CycleTime within target. Using the facilities in the simulator he or she can add additional resource and repeat the simulation and immediately see the effect of that change.

\section{Rules}

Rules are defined in a similar way to KPIs but are more suited to warning conditions or the monitoring of 
regulatory compliance. For example, if the board of this business decide that any jobs with a CycleTime above 500 units should cause an alert condition. A rule can be defined as eduration > 500; and a consequent chosen to perform the desired alert. In this example case on screen alerts were used to present the information required for a process manager to drilldown into the processes and discover the reason for the excessively high CycleTime

Consequents in this scope are actions that the simulator can take when it detects a simulated process breeching the condition. A number of pre-built consequents are available within the simulator however a Java interface is available should a new consequent be required by the user.

\section{Risks}

Risks to a process can be as diverse as the processes themselves and accordingly we have defined a suitably fine-grained approach to the definition of risk within the simulation environment.

Each risk is given a name as an identifier and the probability of occurrence (expressed as a decimal). Each effect of the risk occurring can then be entered using the graphical editor and then saved. The editor itself can be seen in Figure 4.

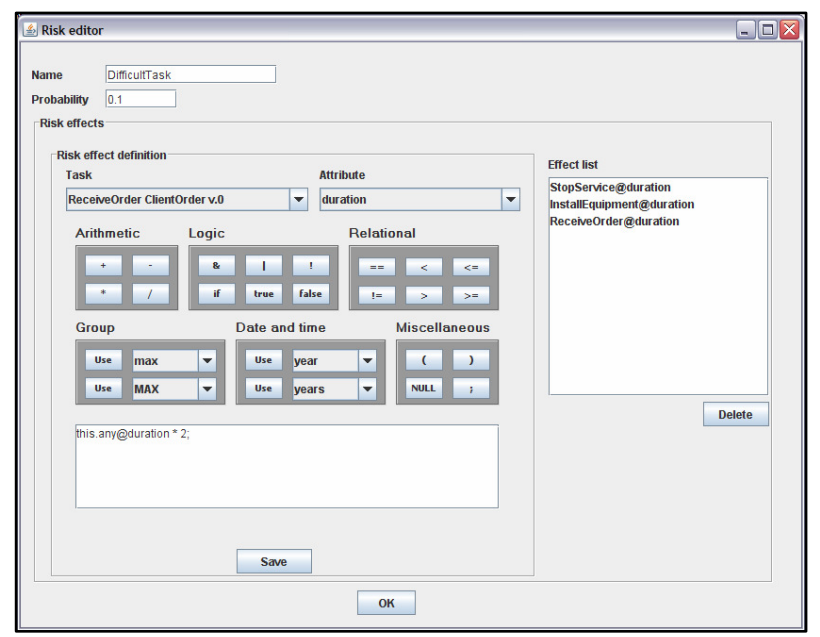

Figure 4: The Risk Editor for the Simulator

Once each risk to the process that needs to be simulated is defined, those to be considered in the current simulation execution can be activated. When activated the simulator will take into account the risk and apply to appropriate processes, allowing the business metrics to be calculated accordingly.

\section{Key Risk Indicators}

As discussed previously KRIs have many resemblances to KPIs but have very different purposes. Consider that this company has performed some risk identification and as a result has defined criteria to judge the risk level of each of the instances of this process (the criteria are given in Table 1). They wish to simulate this risk indicator to gain insight into the current risk level and simulate the effect of changes in the execution of the process, and changes in the execution environment on the processes risk level.

Table 1: Risk Criteria

\begin{tabular}{|c|c|c|}
\hline CycleTime & Cost & Risk Level \\
\hline Short & Low & Low \\
\hline Medium & Low & Low \\
\hline High & Low & Medium \\
\hline Short & High & Medium \\
\hline Medium & High & High \\
\hline High & High & High \\
\hline
\end{tabular}

Given the guidelines and rules used to define what is considered to be Low/Medium/High in each case we can use fuzzy logic in our analysis.

The first step is to use the graphical editor in the simulator to define the fuzzy sets for each of the 3 values (CycleTime, Cost, and Risk Level) using the appropriate functions and values. Once this is completed we assign the fuzzy sets to the attributes they represent (this could be either a task attribute or a process attribute - or both). We then define a fuzzy rule similar to that in Figure 1 as a KRI. The simulator will then use the fuzzy rules that are defined to generate a risk value for each process instance. A graph of these risk values can be seen in Figure 5. For the purposes of this example we have defined the Risk Value to sit within the range $0-100$.

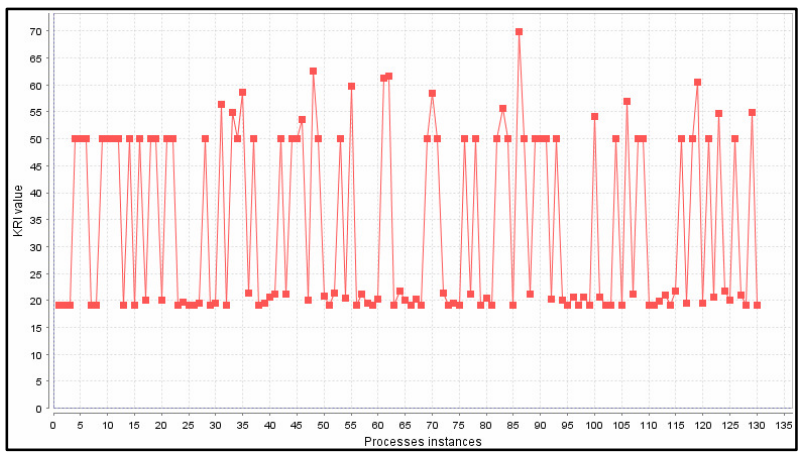

Figure 5: A Graph Showing the Application of the Fuzzy Rule Set,

Additionally the resulting fuzzy set for Risk Value can be displayed with its Centre of Gravity (Figure 6).

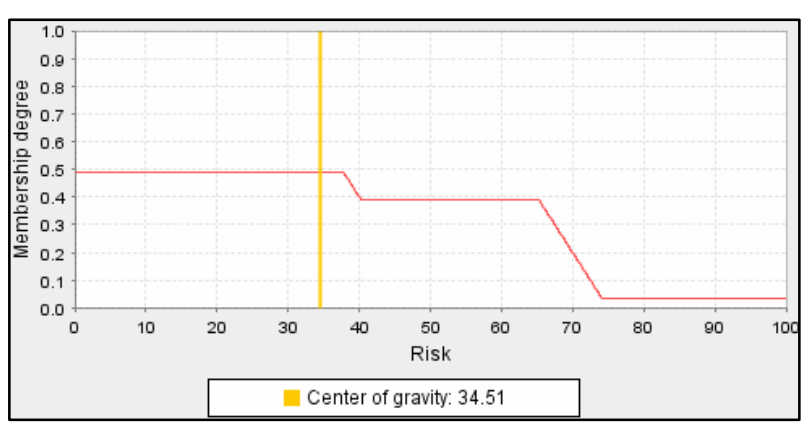

Figure 6: Fuzzy Set for Risk Value 
This shows for each risk value, what membership value that risk would receive. Also the transition points between Low, Medium, and High can be deduced from the shape of the graph. The centre of gravity shows the mean risk.

\section{CONCLUSIONS}

The COSO framework defines Enterprise Risk Management as “... a process [...], applied in strategy setting and across the enterprise, designed to identify potential events that may affect the entity, and manage risk to be within its risk appetite, to provide reasonable assurance regarding the achievement of entity objectives" (COSO, 2004).

This expounds the link between risk analysis and process performance. Therefore it seems clear that to maximize the business value of a process simulation environment, it should be possible (as shown in this paper) to integrate facilities for the performance analysis and monitoring of the process under test into the simulation environment.

Many experts are of the opinion that, for large businesses, qualitative analysis provides the most meaningful risk analysis, however we believe that quantitative risk analysis can provide clear business benefits when used in conjunction with a holistic performance framework such as our RTBI system (see Azvine et al. (2006) for more information).

In this paper we have shown how the business process simulator could be used in the simulation of business processes. We have also shown how risk events, and additionally how fuzzy logic can be utilised in this simulation framework to increase the business utility of the tool.

Furthermore, we have demonstrated how the use of fuzzy logic can help business users express qualitative measures that may change the outcome of the process and gain a quantitative insight into the estimated performance of the process, simplifying the sensitivity analysis of risks.

Since fuzzy logic provides a close analogue for human reasoning (Zadeh 1994) it allows natural expression of sometimes complex business conditions in an intuitive way, whilst preserving the ability to provide quantitative results thus lowering one of the boundaries of adoption for this tool in business.

In summary, it is important for the simulation of risk events in business processes to take place in an integrated framework that will allow performance analysis and risk analysis to be carried out side by side.

\section{REFERENCES}

Azvine, B.; Z. Cui; D.D. Nauck; B. Majeed, "Real Time Business Intelligence for the Adaptive Enterprise", In $E$ Commerce Technology. The 8th IEEE International Conference on Enterprise Computing, E-Commerce, and EServices, pp.29-29, 2006

Azvine, B; Z. Cui; B. Majeed; M. Spott, "Operational Risk Management with Real-Time Business Intelligence", in BT Technology Journal, Vol. 25 No. 1, pp 154-167, 2007.
Bohn, C.; B. Kemp, "Enterprise Risk Management Quantification - An Opportunity". Presented at Enterprise Risk Management Symposium, Society of Actuaries, Chicago, IL, April 23-26, 2006.

COSO, "Enterprise Risk Management - Integrated Framework", 2004.

Davenport, T.H., "Process Innovation: Reengineering Work through Information Technology", Harvard Business School Press, Boston, 1993.

Ken McKinley, "Qualitative and Quantitative Risk Analysis", Project Decision and Risk Analysis Journal, 2005.

Jallow, A.K.; B. Majeed; K. Vergidis; A. Tiwari; R. Roy, "Operational risk analysis in business processes", in BT Technology Journal, Vol. 25, No 1, pp 168-177, 2007.

Lambert, J.H..; R.K. Jennings; N.N. Joshi, "Integration of Risk Identification with Business Process Models", In Systems Engineering, Vol. 9 No. 3, 2006.

Mestchian, P., Risk Intelligence - "From Compliance to Performance", In Journal of Risk Intelligence, Issue 1.

Neiger, D., L. Churilov, M. zur Muehlen, M. Rosemann, "Integrating Risks in Business Process Models with Value Focused Process Engineering”, In European Conference on Information Systems (ECIS), 2006.

Zadeh, L.A., "Soft computing and fuzzy logic", Software, IEEE, Vol.11 No. 6, pp.48-56, 1994.

\section{AUTHOR BIOGRAPHIES}

Paul Taylor joined BT in October 2007 and currently works as a Researcher within the Intelligent Systems Research Centre. He holds a Degree in Computer Science (2006) and a Masters Degree in Computer Security (2007), both from the University of Birmingham. He has previously worked in areas such as Electronic Voting and Protocol Verification and is currently working on the Business Process Management project as part of the Real-Time Business Intelligence theme in BT.

Jesús Jimenez Godino joined BT in October 2005 and currently works as a Researcher within the Intelligent Systems Research Centre. He holds a Masters Degree in Computer Science from the University of Sevilla (2006). He is currently working on the Business Process Management project as part of the Real-Time Business Intelligence theme in BT.

Basim Majeed is a Principal Research Professional at the Intelligent Systems Research Centre within BT Research and Venturing. He holds a Masters degree (1987) and a PhD degree (1992) from the University of Manchester. He is a Member of the IET and IEEE, and a Chartered Engineer. He has published many papers in the areas of intelligent control, fault detection and isolation in dynamic systems, signal processing and intelligent data analysis. Since joining BT in 2003, he worked on a number of projects including the intelligent data analysis project within the Care in the Community Centre and the business process management project within the Real Time Business Intelligence (RTBI) theme. 